\title{
EFEITOS DA POSIÇÃO DE JOGO NA APTIDÃO FÍSICA DE COMPETIDORES AMADORES DE RUGBY UNION
}

\author{
Camila Borges Müller \\ Universidade Federal de Pelotas, Pelotas, Rio Grande do Sul, Brasil \\ Eraldo dos Santos Pinheiro \\ Universidade Federal de Pelotas, Pelotas, Rio Grande do Sul, Brasil \\ Tairã Gonçalves Soares \\ Universidade Federal de Pelotas, Pelotas, Rio Grande do Sul, Brasil \\ Fabricio Boscolo Del Vecchio \\ Universidade Federal de Pelotas, Pelotas, Rio Grande do Sul, Brasil
}

\begin{abstract}
Resumo
Objetivou-se mensurar e correlacionar variáveis antropométricas e de aptidão física de jogadores de rugby union (RU) amadores, segundo posição de jogo nos diferentes sexos. Para isto, 58 jogadores de RU (34 homens e 24 mulheres) foram envolvidos. Aferiram-se estatura (EST), massa corporal (MC), potência de membros inferiores (SJ e CMJ), velocidade de $s$ print de 10m (S10) e 20m (S20), potência anaeróbia (RAST) e resistência anaeróbia (M5). A análise do sexo masculino mostrou que houve diferenças significativas entre posições em EST, MC, SJ, CMJ, S10 e S20, potência de pico, potência relativa à massa corporal e à velocidade do RAST. No sexo feminino, apenas MC variou entre posições. Observaram-se correlações entre S10 e potência média (PM) no sexo masculino, e entre EST e S20, MC e PM, e MC e IF no sexo feminino. Conclui-se que as diferenças na aptidão física entre posições são mais visíveis no sexo masculino, sendo que jogadores da posição backs são mais baixos, leves, rápidos e potentes em relação às demais posições.
\end{abstract}

Palavras-chave: Desempenho atlético. Exercício. Esportes

\section{EFFECTS OF GAME POSITION IN PHYSICAL FITNESS OF RUGBY UNION AM- ATEUR COMPETITORS}

\begin{abstract}
The objective was to measure and correlate anthropometric and physical fitness variables of rugby union (RU) players, according to game position in different sexes, for better characterization of amateur players. $58 \mathrm{RU}$ players (34 men and 24 women) were involved. Stature (EST), body mass (MC), lower limb power (SJ and CMJ), 10m (S10) and 20m (S20) speed, anaerobic power (RAST), and anaerobic resistance (M5). Among men, there were significant differences between the emissions in EST, MC, SJ, CMJ, S10 and S20, peak power, power relative to body mass and RAST velocity. In the female, only MC ranged between families. There were correlations between S10 and mean power (PM) in males, and between EST and S20, MC and PM, and MC and IF in female. It follows that physics passive nations are the most visible in men and players in the rear position are lower, lighter, faster and more powerful.
\end{abstract}

Keywords: Athletic performance. Exercise. Sports. 


\section{EFECTOS DE LA POSICIÓN DE JUEGO EN LA APTITUD FÍSICA DE COMPETI- DORES AFICIONADOS DE RUGBY UNION}

\section{Resumen}

Se objetivó medir y correlacionar variables antropométricas y de aptitud física de jugadores de rugby union (RU), según posición de juego, para una mejor caracterización de jugadores aficionados. 58 jugadores de RU (34 hombres y 24 mujeres) fueron involucrados. Se midieron estatura (EST), masa corporal (MC), potencia de miembros inferiores (SJ y CMJ), velocidad de sprint de $10 \mathrm{~m}$ (S10) y 20m (S20), potencia anaerobia (RAST), y resistencia anaerobia (M5). Entre hombres, hubo diferencias significativas entre posiciones en EST, MC, SJ, CMJ, S10 y S20, potencia de pico, potencia relativa a la masa corporal y velocidad del RAST. En el femenino, sólo MC varió entre posiciones. Se observaron correlaciones entre S10 y potencia media (PM) en el masculino, y entre EST y S20, MC y PM, y MC e IF en el femenino. Se concluye que las diferencias em la aptitud física entre posiciones son más visibles em los hombres, y los jugadores de la posición backs son más bajos, ligeiros, rápidos y potentes.

Palabras clave: Desempeño atlético. Ejercicio. Deportes

\section{Introdução}

As exigências físicas do rugby union são caracterizadas como frequentes esforços intermitentes com presença de contatos físicos estáticos e dinâmicos e curtos momentos de baixa intensidade ou de repouso (DUTHIE; PYNE; HOOPER, 2003; CUNNINGHAM et al., 2016). Devido a essa característica intermitente, jogadores de rugby realizam sucessivos esforços de 5 a 15 segundos de duração com até 40 segundos de recuperação entre eles (NICHOLAS, 1997; FULLER et al., 2007). Nesse contexto, a potência e a velocidade são capacidades fundamentais para o desempenho no rugby (DUTHIE; PYNE; HOOPER, 2003; HAFF; NIMPHIUS, 2012). A potência muscular desenvolvida aumenta a produção de força e a velocidade de aceleração, podendo melhorar a capacidade global do atleta (HAFF; NIMPHIUS, 2012). No rugby, a capacidade de aceleração nos sprints é essencial para atingir o objetivo do jogo, visto que a duração média de sprints nos jogos é de aproximadamente 3 segundos e, nesse contexto, níveis superiores de potência são primordiais para atletas da modalidade (DUTHIE, 2006).

Dessa forma, o conhecimento das características fisiológicas, neuromotoras e das demandas do jogo deve ser considerado por treinadores na prescrição do treinamento e por pesquisadores na análise das variáveis envolvidas. Aponta-se que as capacidades físicas são determinantes para discriminar jogadores de diferentes níveis (BAKER, 2017) e que variáveis como potência, agilidade e resistência anaeróbia são essenciais na avaliação de jogadores de rugby, além de auxiliarem treinadores na identificação de pontos fracos e fortes de seus times (ROE et al., 2016).

No rugby union, existem duas posições específicas de acordo com as demandas do jogo: forwards, que estão mais envolvidos em colisões com os adversários, e backs, que percorrem distâncias maiores durante o jogo (CUNNINGHAM et al., 2016). Adicionalmente, backs tendem a ser mais ágeis e velozes que forwards (DUTHIE, 2006), uma vez que backs externos tendem a se especializar nos aspectos de funcionamento do jogo, enquanto backs internos se envolvem em aspectos de confronto como rucks, mauls e tackles (DEUTSCH; KEARNEY; REHRER, 2007) e forwards da primeira linha parecem ter maior envolvimento em ganhar e manter a posse de bola, realizando com frequência rucks e mauls; já forwards da segunda e 
terceira linha desempenham o papel de quebra de jogo, como sprints e tackles (JONES et al. 2015).

De modo amplo, a literatura reforça diferenças entre forwards e backs no que se refere a características físicas e antropométricas (DUTHIE; PYNE; HOOPER, 2003; CUNNINGHAM et al., 2016), sendo que os primeiros tendem a ser mais altos e mais pesados, têm maior percentual de gordura corporal, bem como potência aeróbia e anaeróbia absolutas e força muscular superior aos backs (DUTHIE; PYNE; HOOPER, 2003). Jogadores da seleção brasileira de rugby union têm aptidão física semelhante à encontrada no cenário internacional, bem como foram observadas diferenças entre forwards e backs (NAKAMURA et al., 2016). Em contrapartida, medidas de aptidão física de jogadores amadores, com comparações entre posições e sexos no Brasil, são incomuns (PINHEIRO et al., 2013).

Nesse sentido, investigar a relação entre variáveis antropométricas e a aptidão física de jogadores amadores de rugby pode ser relevante para melhor caracterização das posições de jogo (HOHENAUER et al., 2017; WOOD; COUGHLAN; DELAHUNT, 2018; COMFORT et al., 2011), bem como identificar o perfil de jogadores brasileiros de rugby union. Complementarmente, mensurar a aptidão física para realizar treinamento adequado é fator relevante para o incremento do desempenho físico. Portanto, o objetivo do presente estudo foi mensurar e correlacionar as variáveis de aptidão física de jogadores de rugby union amadores dos diferentes sexos, segundo posições de jogo.

\section{Métodos}

O estudo, observacional e de comparação, envolveu 58 atletas amadores de rugby union (34 homens e 24 mulheres), de clubes da região sul do Rio Grande do Sul, que disputaram campeonatos de nível estadual. Os participantes tinham entre 15 e 36 anos, com no mínimo dois anos de prática regular da modalidade. Foram excluídos da amostra jogadores com doenças reconhecidas e diagnosticadas, lesionados nos últimos três meses e em recuperação, que faziam uso de recursos ergogênicos farmacológicos e medicamentos de uso contínuo. A aplicação da bateria de testes foi precedida por leitura e anuência a partir de assinatura do termo de consentimento livre e esclarecido (Projeto aprovado no COCEPE: 52291056/2016).

$\mathrm{O}$ recrutamento da amostra se deu por convite verbal aos jogadores de duas equipes amadoras de rugby do Rio Grande do Sul. Realizou-se o agendamento da avaliação, e os participantes voluntários que respeitavam os critérios de inclusão foram recrutados para as avaliações. Quanto ao período competitivo, destaca-se que todos os participantes estavam em temporada de competições. Solicitou-se que os participantes se privassem de esforços vigorosos $48 \mathrm{~h}$ antes do dia agendado para as coletas, evitassem consumo de álcool e de bebidas cafeinadas nas $24 \mathrm{~h}$ anteriores. Também foi indicado que eles dormissem por, pelo menos, $8 \mathrm{~h}$ na noite anterior ao dia das coletas.

As atividades ocorreram em campo com grama natural, no período de $9 \mathrm{~h}$ a $12 \mathrm{~h}$, com temperatura entre $21^{\circ} \mathrm{C}$ e $25^{\circ} \mathrm{C}$. Para cada participante, os procedimentos foram realizados em sessão única, por grupo de avaliadores previamente treinados, e sob supervisão contínua e proximal. As variáveis antropométricas medidas foram estatura (EST) e massa corporal (MC). A aptidão física dos atletas foi avaliada por meio de testes físicos de velocidade, potência e resistência, nesta ordem, com intervalo entre testes de, pelo menos, 15 minutos. A velocidade foi medida por teste de sprints, com registro do tempo em 10m e 20m (MOIR et al., 2004); a potência de membros inferiores foi mensurada a partir de saltos verticais (MARKOVIC et al., 2004); a potência e a capacidade anaeróbias foram avaliadas com o Running-based anaerobic sprint test (RAST) (ZAGATTO; BECK; GOBATTO, 2009), e a resistência anaeróbia pelo teste múltiplo de 5 (M5) (BODDINGTON et al., 2001). 
Para avaliar a velocidade de sprints de $10 \mathrm{~m}$ (S10) e $20 \mathrm{~m}$ (S20), foram posicionadas fotocélulas (Multisprint, Hidrofit@) a 10 e a 20 metros após o ponto de partida. Os indivíduos se deslocaram do local de saída até a última fotocélula na maior velocidade possível, registrando o tempo atingido nas distâncias demarcadas. Os sujeitos realizaram três corridas máximas, com intervalo passivo de quatro minutos entre as tentativas, e foi registrado o menor tempo dispendido para percorrer os 10 e $20 \mathrm{~m}$. Ainda, os jogadores foram orientados a não diminuírem a velocidade até ultrapassarem a última fotocélula com a finalidade de o teste ser realizado em aceleração máxima. $\mathrm{O}$ teste de velocidade tem reprodutibilidade teste-reteste de $\mathrm{r}=$ 0,89 (MOIR et al., 2004).

A potência de membros inferiores foi avaliada a partir de dois tipos de saltos verticais: Squat Jump (SJ) e Countermovement Jump (CMJ). Os procedimentos foram realizados sobre tapete de contato (Jump System 1.0®, CEFISE, Nova Odessa, Brasil), de acordo com o protocolo sugerido por Markovic et al. (2004). Para cada tipo de salto, foram realizadas três tentativas com intervalo de quatro minutos e foi registrada a melhor marca. O teste de SJ consistiu em partir de uma flexão de $90^{\circ}$ dos joelhos, checada com goniômetro, com as mãos na cintura e, após três segundos, impulsionar-se para cima com a maior velocidade e força possíveis. Não foi permitido utilizar a flexão dos joelhos, do quadril e o auxílio dos braços durante o tempo de voo. As tentativas que apresentaram erros foram desconsideradas e repetidas, e este procedimento tem reprodutibilidade teste-reteste de $r=0,93$ (MARKOVIC et al., 2004). O CMJ foi avaliado partindo da posição em pé e com mãos na cintura, em que o sujeito deveria realizar um agachamento de aproximadamente $90^{\circ} \mathrm{e}, \operatorname{logo}$ após, impulsão vertical o mais alto possível sem o auxílio dos membros superiores (reprodutibilidade teste-reteste de $r=0,93$ ) (MARKOVIC et al., 2004). A partir da diferença entre o CMJ e o SJ, obteve-se o "índice de salto", sendo que valores inferiores a $3 \mathrm{~cm}$ foram considerados como "fraco", entre 3 e $6 \mathrm{~cm}$ como "regular" e superiores a $6 \mathrm{~cm}$ como "superior", conforme o sugerido por Gerodimos et al. (2008).

Para avaliação da potência anaeróbia, o RAST foi aplicado a partir do seguinte procedimento: seis sprints de $35 \mathrm{~m}$ na maior velocidade possível, com intervalo passivo de $10 \mathrm{~s}$. A distância foi marcada por cones e foram posicionadas duas fotocélulas para registrar a velocidade de cada sprint atingida pelos sujeitos. Posteriormente, foi calculada a potência em watts (W) pela equação (Massa Corporal x Distância²)/Tempo ${ }^{3}$. Além disso, foi calculado o índice de fadiga (IF) através da equação ((potência máxima - potência mínima)/potência máxima). $\mathrm{O}$ RAST conta com reprodutibilidade teste-reteste de $r=0,97$ (ZAGATTO et al., 2009).

A resistência anaeróbia geral foi avaliada através do teste Múltiplo de 5 (M5), que consiste em percorrer trajeto de 25 metros divididos por seis cones dispostos a cada 5 metros (Figura 1), que demarcavam as linhas a serem atingidas. Os sujeitos foram instruídos a percorrer a maior distância possível durante todo o teste. A partir de um sinal auditivo, os jogadores partiram do primeiro cone até o segundo cone a $5 \mathrm{~m}$, tocando no solo na linha correspondente e voltando para o primeiro cone, novamente tocando com uma das mãos no chão em cima da linha de partida. Posteriormente, eles correram $10 \mathrm{~m}$ até o terceiro cone, realizando o mesmo procedimento de volta para o início, e assim progredindo sucessivamente durante 30 s. Foram realizadas seis séries máximas de $30 \mathrm{~s}$, com um intervalo passivo de $35 \mathrm{~s}$, e cada série foi contabilizada pela distância percorrida. Ao final do teste, foi registrada a distância total percorrida pelos sujeitos. O teste apresenta coeficiente de correlação intraclasse de 0,98 para distância total (BODDINGTON et al., 2001). 
Figura 1. Esquema gráfico da execução do Teste Múltiplo de 5

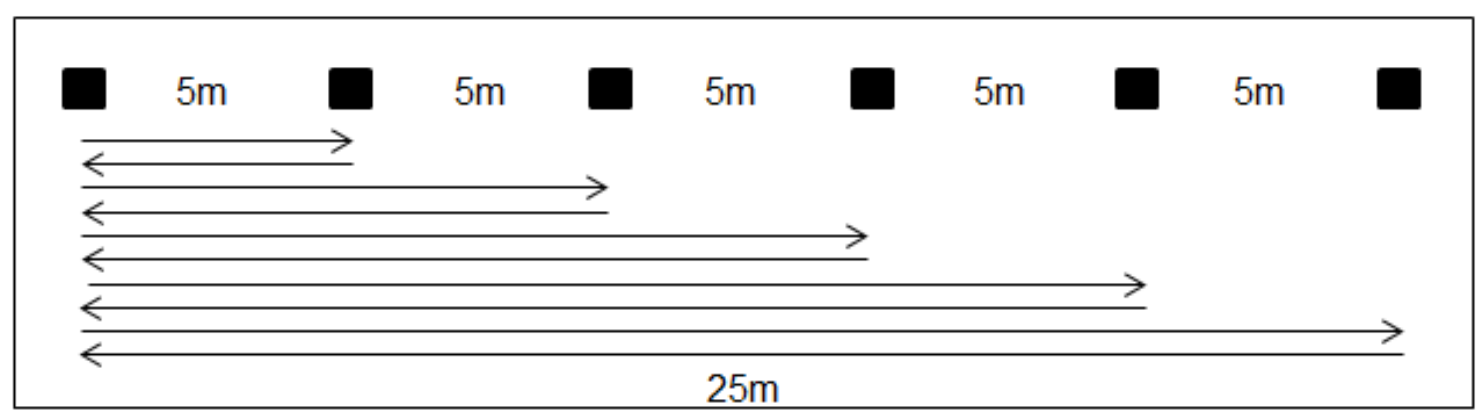

Todas as variáveis foram testadas quanto à distribuição por meio do teste de ShapiroWilk. Após confirmar a distribuição normal dos dados, foram utilizados os seguintes procedimentos: a) para apresentação dos resultados descritivos, utilizaram-se média e desvio padrão; b) para comparação entre sexos em relação às características pessoais, empregou-se o teste $t$ para amostras não pareadas; c) na comparação entre backs e forwards, bem como entre sexos, foi utilizada uma ANOVA two-way, com post-hoc de Tukey; d) para o índice de salto, foi utilizada a diferença entre o CMJ e o SJ classificadas por $<3=$ fraco, entre 3 e $6=$ regular e $>6$ ótimo; e) as correlações entre as medidas dos testes de aptidão física foram avaliadas com teste de correlação de Pearson. Foi realizado cálculo do tamanho do efeito (TE), quando adequado a partir do $\mathrm{d}$ de Cohen e subsequente classificação como trivial $(<0,35)$, pequena $(0,35-0,80$, moderada $(0,80-1,50)$ ou grande $(>1,50)$ (RHEA, 2004). Depois, realizou-se regressão linear para verificar os efeitos de variáveis de interesse no desempenho no teste múltiplo de 5. Para todas as análises, foi utilizado o pacote estatístico SPSS 20.0.

\section{Resultados}

Os sujeitos apresentaram 24,57 \pm 4,97 anos (mulheres $=22,92 \pm 2,81$ e homens $=$ $25,74 \pm 5,81, \mathrm{t}=2,46 ; \mathrm{p}=0,017)$, com estatura de $1,70 \pm 0,09 \mathrm{~m}$ e massa corporal média de $83,29 \pm 23,87 \mathrm{~kg}$. A Tabela 1 apresenta a comparação dos forwards e dos backs em cada sexo. Para a estatura, foram observadas diferenças entre posições apenas no sexo masculino $(\mathrm{F}=8,36 ; \mathrm{p}<0,01 ; \mathrm{TE}=0,92)$. Para a massa corporal, foram observadas diferenças significativas entre posições nos homens $(\mathrm{F}=30,50 ; \mathrm{p}<0,01 ; \mathrm{TE}=1,86)$ e nas mulheres $(\mathrm{F}=25,56 ; \mathrm{p}<0,01$; $\mathrm{TE}=1,99)$. Foram observadas diferenças significativas apenas no sexo masculino em ambos os saltos $\mathrm{SJ}(\mathrm{F}=8,86 ; \mathrm{p}<0,01 ; \mathrm{TE}=1,00)$ e $\mathrm{CMJ}(\mathrm{F}=10,46 ; \mathrm{p}<0,01 ; \mathrm{TE}=1,10)$, bem como nas velocidades $\mathrm{S} 10(\mathrm{~F}=6,56 ; \mathrm{p}=0,01 ; \mathrm{TE}=0,92)$ e $\mathrm{S} 20(\mathrm{~F}=6,53 ; \mathrm{p}=0,02 ; \mathrm{TE}=0,82)$. Para o M5, não houve diferença significativa entre posições. Nos valores relacionados ao RAST (Tabela 2), foram observadas diferenças significativas entre forwards e backs apenas nos homens nas variáveis potência de pico $(\mathrm{F}=5,49 ; \mathrm{p}<0,01 ; \mathrm{TE}=0,821)$ e menor velocidade no RAST $(\mathrm{F}=9,17 ; \mathrm{p}<0,01 ; \mathrm{TE}=0,37)$.

Quanto ao índice de salto, os homens apresentaram diferença entre as médias de CMJ e SJ de $0,99 \mathrm{~cm}$ para os forwards e $2,01 \mathrm{~cm}$ para os backs $(\mathrm{t}=3,66 ; \mathrm{p}<0,01)$. As classificações de desempenho do índice de salto mostram que, no sexo masculino, $81 \%$ foram considerados "fracos" $(<3 \mathrm{~cm}), 8,7 \%$ foram "regulares" $(3-6 \mathrm{~cm})$ e 10,3\% foram "ótimos" ( $>6 \mathrm{~cm})$. Nas mulheres, forwards apresentaram diferença média de $1,54 \mathrm{~cm}$, enquanto as backs apresentaram 2,57 cm $(\mathrm{t}=3,66 ; \mathrm{p}<0,01)$ e, nas classificações de desempenho, 75\% foram consideradas "fracas", 12,5\% foram "regulares" e 12,5\% foram "ótimas".

Nas correlações das variáveis analisadas (Tabela 3), destaca-se que houve correlação moderada e significante nos homens entre sprint $10 \mathrm{~m}$ e potência média $(\mathrm{r}=-0,612)$ e, nas mulheres, entre estatura e sprint $20 \mathrm{~m}(\mathrm{r}=-0,652)$, massa corporal e potência média $(\mathrm{r}=0,650)$, 
bem como massa corporal e índice de fadiga $(\mathrm{r}=0,645)$.

A partir da análise de regressão linear, observou-se que a potência relativa à massa corporal no RAST e o CMJ foram as únicas variáveis que explicaram a distância percorrida no teste Múltiplo de 5. A Tabela 5 apresenta a regressão linear do Múltiplo de 5, a qual é quase $50 \%$ determinada pela potência relativa à massa corporal no sexo masculino e em mais de $20 \%$ pelo CMJ no sexo feminino.

Tabela 1. Diferenças antropométricas e na aptidão física entre posições nos diferentes sexos

\begin{tabular}{|c|c|c|c|c|c|c|c|c|}
\hline \multirow{2}{*}{$\begin{array}{l}\text { Variá- } \\
\text { vel } \\
\text { (un) }\end{array}$} & \multicolumn{3}{|c|}{ Masculino } & \multicolumn{3}{|c|}{ Feminino } & \multirow{2}{*}{$\begin{array}{l}\begin{array}{l}\text { Posi- } \\
\text { ção }\end{array} \\
\text { F (p) }\end{array}$} & \multirow{2}{*}{$\begin{array}{l}\begin{array}{l}\text { Intera- } \\
\text { ção }\end{array} \\
\mathrm{F}(\mathrm{p})\end{array}$} \\
\hline & Forwards & Backs & Todos & Forwards & Backs & Todas & & \\
\hline $\begin{array}{l}E S T \\
(m)\end{array}$ & $1,79 \pm 0,07$ & $\begin{array}{c}1,73 \pm 0,06 \\
*\end{array}$ & $1,76 \pm 0,07$ & $1,66 \pm 0,09$ & $1,61 \pm 0,05$ & $\begin{array}{l}1,63 \pm 0,0 \\
7\end{array}$ & $\begin{array}{l}10,19 \\
(<0,01)\end{array}$ & $\begin{array}{l}0,13 \\
(0,72)\end{array}$ \\
\hline $\begin{array}{l}M C \\
(k g)\end{array}$ & $\begin{array}{c}111,96 \pm 22 \\
74\end{array}$ & $\begin{array}{c}77,43 \pm 12,9 \\
2 *\end{array}$ & $\begin{array}{l}93,68 \pm 25,0 \\
4\end{array}$ & $79,72 \pm 10,91$ & $\begin{array}{l}62,23 \pm 5,85 \\
*\end{array}$ & $\begin{array}{l}68,32 \pm 11 \\
, 5\end{array}$ & $\begin{array}{l}38,89 \\
(<0,01)\end{array}$ & $\begin{array}{l}4,17 \\
(0,05)\end{array}$ \\
\hline $\begin{array}{c}S J \\
(\mathrm{~cm})\end{array}$ & $29 \pm 8,67$ & $\begin{array}{c}35,95 \pm 4,5 \\
5^{*}\end{array}$ & $32,68 \pm 7,57$ & $21,02 \pm 3,42$ & $24,07 \pm 5,55$ & $\begin{array}{l}23,01 \pm 5 \\
05\end{array}$ & $\begin{array}{l}8,59 \\
(<0,01)\end{array}$ & $\begin{array}{l}1,31 \\
(0,26)\end{array}$ \\
\hline $\begin{array}{l}C M J \\
(\mathrm{~cm})\end{array}$ & $30 \pm 8,76$ & $\begin{array}{c}37,96 \pm 5,4 \\
1^{*}\end{array}$ & $34,21 \pm 8,14$ & $22,76 \pm 9,05$ & $26,64 \pm 5,07$ & $\begin{array}{l}25,29 \pm 6 \\
78\end{array}$ & $\begin{array}{l}9,31 \\
(<0,01)\end{array}$ & $\begin{array}{l}1,11 \\
(0,3)\end{array}$ \\
\hline $\begin{array}{c}\text { S10m } \\
(s)\end{array}$ & $2,04 \pm 0,16$ & $\begin{array}{c}1,91 \pm 0,12 \\
*\end{array}$ & $1,97 \pm 9,15$ & $2,19 \pm 0,17$ & $2,15 \pm 0,12$ & $\begin{array}{l}2,16 \pm 0,1 \\
4\end{array}$ & $\begin{array}{l}4,62 \\
(0,04)\end{array}$ & $\begin{array}{l}1,06 \\
(0,31)\end{array}$ \\
\hline $\begin{array}{c}S 20 m \\
(s)\end{array}$ & $3,51 \pm 0,25$ & $\begin{array}{c}3,33 \pm 0,18 \\
*\end{array}$ & $3,41 \pm 0,23$ & $3,96 \pm 0,22$ & $3,89 \pm 0,16$ & $\begin{array}{l}3,90 \pm \\
0,18\end{array}$ & $\begin{array}{l}6,11 \\
(0,02)\end{array}$ & $\begin{array}{l}0,76 \\
(0,39)\end{array}$ \\
\hline$M 5(m)$ & $\begin{array}{c}532,81 \pm 92 \\
99\end{array}$ & $585 \pm 73,60$ & $\begin{array}{l}560,44 \pm 86, \\
14\end{array}$ & $\begin{array}{l}438,75 \pm 107 \\
89\end{array}$ & $\begin{array}{l}486,67 \pm 76 \\
03\end{array}$ & $\begin{array}{l}470 \pm 89,0 \\
3\end{array}$ & $\begin{array}{l}4,46 \\
(0,04)\end{array}$ & $\begin{array}{l}0,01 \\
(0,93)\end{array}$ \\
\hline
\end{tabular}

Fonte: Elaborado pelos autores, 2018.

$\mathrm{EST}=$ Estatura MC = Massa Corporal; $\mathrm{SJ}=$ Squat Jump; $\mathrm{CMJ}=$ Countermovement Jump; M5 = Múltiplo de 5; $\mathrm{S} 10=$ Sprint $10 \mathrm{~m} ; \mathrm{S} 20=$ Sprint $20 \mathrm{~m} . *$ Estatisticamente diferente dos forwards $(\mathrm{p}<0,05)$

Tabela 2. Valores de potência, velocidade mínima e índice de fadiga relacionados ao RAST

\begin{tabular}{|c|c|c|c|c|c|c|c|c|}
\hline \multirow{2}{*}{$\begin{array}{l}\text { Variável } \\
\text { (un) }\end{array}$} & \multicolumn{3}{|c|}{ Masculino } & \multicolumn{3}{|c|}{ Feminino } & \multirow{2}{*}{$\begin{array}{c}\text { Posição } \\
F(p)\end{array}$} & \multirow{2}{*}{$\begin{array}{l}\begin{array}{l}\text { Inte- } \\
\text { ração }\end{array} \\
\mathrm{F}(\mathrm{p})\end{array}$} \\
\hline & $\begin{array}{l}\text { For- } \\
\text { wards }\end{array}$ & Backs & Todos & $\begin{array}{l}\text { For- } \\
\text { wards }\end{array}$ & Backs & Todos & & \\
\hline$P M(W)$ & $\begin{array}{c}498,9 \pm 83, \\
89\end{array}$ & $\begin{array}{c}458,26 \pm 79,5 \\
9\end{array}$ & $\begin{array}{l}477,34 \pm 82 \\
, 93\end{array}$ & $\begin{array}{l}245,53 \pm 79 \\
, 82\end{array}$ & $\begin{array}{l}221,1 \pm 46, \\
11\end{array}$ & $\begin{array}{l}230,26 \pm 60 \\
, 47\end{array}$ & $\begin{array}{l}2,56 \\
(0,11)\end{array}$ & $\begin{array}{l}0,16 \\
(0,69)\end{array}$ \\
\hline$P P(W)$ & $\begin{array}{c}722,34 \pm 1 \\
38\end{array}$ & $\begin{array}{c}614,41 \pm 124 \\
71^{*}\end{array}$ & $\begin{array}{l}666,63 \pm 14 \\
0,25\end{array}$ & $\begin{array}{l}332,28 \pm 92 \\
, 48\end{array}$ & $\begin{array}{l}297,5 \pm 58, \\
65\end{array}$ & $\begin{array}{l}310,54 \pm 73 \\
, 24\end{array}$ & $\begin{array}{l}5,49 \\
(<0,01)\end{array}$ & $\begin{array}{l}1,44 \\
(0,23)\end{array}$ \\
\hline $\begin{array}{l}\text { PRelMC } \\
(\mathrm{W} / \mathrm{kg})\end{array}$ & $4,68 \pm 1,25$ & $5,87 \pm 0,90^{*}$ & $5,29 \pm 1,22$ & $3,06 \pm 0,61$ & $3,56 \pm 0,70$ & $3,37 \pm 0,7$ & $\begin{array}{l}10,89 \\
(<0,01)\end{array}$ & $\begin{array}{l}1,78 \\
(0,89)\end{array}$ \\
\hline $\operatorname{VRAST}(s)$ & $5,75 \pm 0,49$ & $5,41 \pm 1,22 *$ & $5,58 \pm 0,41$ & $6,69 \pm 0,35$ & $6,39 \pm 0,39$ & $6,5 \pm 0,4$ & $\begin{array}{l}9,17 \\
(<0,01)\end{array}$ & $\begin{array}{l}0,04 \\
(0,84)\end{array}$ \\
\hline$I F(\%)$ & $1,16 \pm 4,45$ & $1,71 \pm 1,72$ & $1,44 \pm 3,29$ & $1,53 \pm 7,68$ & $2,57 \pm 2,47$ & $2,18 \pm 4,95$ & $\begin{array}{l}0,48 \\
(0,49)\end{array}$ & $\begin{array}{l}0,04 \\
(0,84)\end{array}$ \\
\hline
\end{tabular}

Fonte: Elaborado pelos autores, 2018. PM = Potência Média; PP = Potência de Pico; PRelMC = Potência Relativa à Massa Corporal; VRAST = Menor velocidade no RAST; IF = Índice de Fadiga. *Estatisticamente diferente dos forwards $(\mathrm{p}<0,05)$. 
Tabela 3. Coeficientes de correlação (r) das variáveis de aptidão física de jogadores de rugby dos dois sexos

\begin{tabular}{|c|c|c|c|c|c|c|c|c|c|}
\hline Sexo & $\begin{array}{l}\text { Variá- } \\
\text { veis }\end{array}$ & $\begin{array}{l}\mathrm{MC} \\
(\mathrm{kg})\end{array}$ & $\begin{array}{c}\mathrm{SJ} \\
(\mathrm{cm})\end{array}$ & $\begin{array}{l}\text { CMJ } \\
(\mathrm{cm})\end{array}$ & $\begin{array}{l}\text { M5 } \\
\text { (m) }\end{array}$ & $\begin{array}{l}\text { S10 } \\
\text { (s) }\end{array}$ & S20 (s) & $\begin{array}{l}\mathrm{PM} \\
(\mathrm{W})\end{array}$ & IF \\
\hline \multirow{8}{*}{$\begin{array}{c}\text { Masculi- } \\
\text { no }\end{array}$} & $\operatorname{EST}(\mathrm{m})$ & $0,495^{*}$ & $\begin{array}{c}- \\
0,063\end{array}$ & $-0,167$ & $-0,107$ & 0,033 & 0,184 & 0,318 & 0,282 \\
\hline & $\mathrm{MC}(\mathrm{kg})$ & - & 0,118 & 0,187 & $0, \overline{584 *}$ & 0,314 & $0,447 *$ & 0,093 & 0,262 \\
\hline & $\mathrm{SJ}(\mathrm{cm})$ & - & - & $0,893^{*}$ & 0,114 & $\begin{array}{c}- \\
0,450 \\
*\end{array}$ & $\overline{-}, 434 *$ & $0,519^{*}$ & 0,337 \\
\hline & $\begin{array}{l}\text { CMJ } \\
(\mathrm{cm})\end{array}$ & - & - & - & 0,004 & $\begin{array}{c}- \\
0,414 \\
*\end{array}$ & - $0,413 *$ & $0,516^{*}$ & $0,408 *$ \\
\hline & M5 (m) & - & - & - & - & $\begin{array}{c}- \\
0,513 \\
*\end{array}$ & $\stackrel{-}{0,511 *}$ & 0,307 & $-0,086$ \\
\hline & S10 (s) & - & - & - & - & - & $0,947^{*}$ & $0, \overline{-}$ & $\begin{array}{c}- \\
0,426^{*}\end{array}$ \\
\hline & S20 (s) & - & - & - & - & - & - & $\stackrel{-}{-}$ & $\begin{array}{c}- \\
0,394 *\end{array}$ \\
\hline & PM (W) & - & - & - & - & - & - & - & $0,550^{*}$ \\
\hline \multirow{8}{*}{ Feminino } & EST (m) & $0,423 *$ & 0,086 & 0,301 & 0,351 & $\begin{array}{c}0,501 \\
*\end{array}$ & $0,652 *$ & 0,098 & 0,023 \\
\hline & $\mathrm{MC}(\mathrm{kg})$ & - & $\begin{array}{c}- \\
0,079 \\
\end{array}$ & 0,055 & 0,260 & $\begin{array}{c}0,497 \\
*\end{array}$ & $0,465^{*}$ & $0,650 *$ & $0,645^{*}$ \\
\hline & $\mathrm{SJ}(\mathrm{cm})$ & - & - & $0,645^{*}$ & $-0,027$ & $\begin{array}{c}0,547 \\
*\end{array}$ & 0,373 & 0,224 & 0,247 \\
\hline & $\begin{array}{l}\text { CMJ } \\
(\mathrm{cm})\end{array}$ & - & - & - & $0,416^{*}$ & 0,238 & 0,245 & 0,390 & 0,357 \\
\hline & M5 (m) & - & - & - & - & 0,177 & 0,390 & 0,385 & 0,283 \\
\hline & S10 (s) & - & - & - & - & - & $0,876^{*}$ & 0,287 & 0,215 \\
\hline & S20 (s) & - & - & - & - & - & - & 0,239 & 0,165 \\
\hline & PM (W) & - & - & - & - & - & - & - & $0,951 *$ \\
\hline
\end{tabular}

Fonte: Elaborado pelos autores, 2018. EST = Estatura; MC = Massa Corporal; SJ = Squat Jump; CMJ = Countermovement Jump; M5 = Múltiplo de 5; S10 = Sprint 10m; S20 = Sprint 20m; PM = Potência Média. $* \mathrm{p}<0,05$.

Tabela 5. Regressão linear

\begin{tabular}{|c|c|c|c|c|c|}
\hline Variável & Sexo & Equação da regressão & $\mathrm{r}^{2}$ & $\mathrm{~F}$ & $\mathrm{P}$ \\
\hline Distância total & Masculino & PRelMC x $(366,137)+(39,08)$ & 0,498 & 28,715 & $<0,001$ \\
\hline $\begin{array}{l}\text { percorrida no } \\
\text { teste M5 }\end{array}$ & Feminino & CMJ x $(6,02)+(317,74)$ & 0,210 & 5,590 & 0,028 \\
\hline
\end{tabular}

Fonte: Elaborado pelos autores, 2018.

M5 = Múltiplo de 5; PRelMC = Potência Relacionada à Massa Corporal; CMJ = Countermovement Jump.

\section{Discussão}

O presente estudo se propôs a investigar e relacionar as diferenças na aptidão física entre diferentes posições de jogo no rugby union. No sexo masculino, a maioria das variáveis apresentou diferença entre forwards e backs; porém, no sexo feminino, todas as variáveis foram semelhantes entre posições, com exceção à massa corporal. Nesse sentido, destaca-se que 
o comportamento das variáveis da aptidão física é diferente entre sexos, quando comparados por posições. Além disso, 55\% das correlações se mostraram diferentes entre os sexos, explicitando dissimilaridade elevada.

Do ponto de vista demográfico, atletas de elite da seleção brasileira masculina de rugby union exibem idade $(24,4 \pm 4,2$ anos), estatura $(1,79 \pm 6,7 \mathrm{~m})$ e massa corporal $(90 \pm 11,5$ $\mathrm{kg}$ ) semelhantes aos do presente estudo (KOBAL et al., 2016). Ainda, atletas subelite do

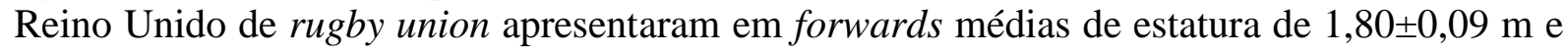

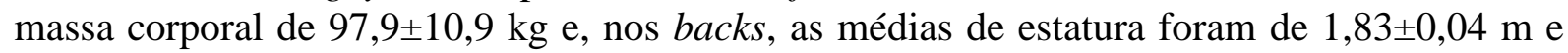
massa corporal de 86,4 $\pm 10,7 \mathrm{~kg}$ (JARVIS et al., 2009). Da mesma forma, o comportamento da massa corporal foi maior em forwards neste estudo, com tamanho do efeito considerado grande. Os forwards da presente investigação apresentaram massa corporal superior aos atletas subelite, provavelmente pelo maior percentual de gordura corporal, o que pode influenciar sobremaneira no desempenho esportivo, tendo em vista que os forwards mais pesados podem apresentar uma quantidade de massa não contrátil maior. Além disso, os backs do presente estudo apresentaram estatura e massa corporal inferiores aos atletas de subelite, talvez pelo nível competitivo desses jogadores ser maior. Já em jogadores amadores de rugby union,

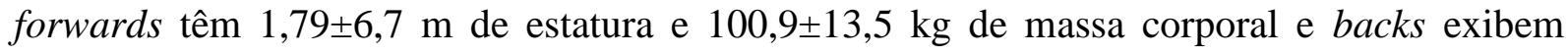
$1,80 \pm 7,6 \mathrm{~m}$ de estatura e $81,6 \pm 7,2 \mathrm{~kg}$ de massa corporal (SANT'ANNA, 2015), resultados semelhantes ao presente estudo, considerando-se a semelhança entre os grupos avaliados nos dois estudos, que remete a uma parte importante da realidade do rugby brasileiro.

No sexo feminino, forwards têm 1,66 m de estatura e 75,6 kg de massa corporal e backs têm estatura semelhante $(1,64 \mathrm{~m})$, mas massa corporal inferior $(61,4 \mathrm{~kg})$ (QUARRIE et al., 1995). Atletas de elite canadenses foram investigadas nas variáveis antropométricas e apresentaram $1,70 \pm 4,3 \mathrm{~m}$ e $1,66 \pm 6,0 \mathrm{~m}$ e massa corporal de 72,87 $\pm 4,79 \mathrm{~kg}$ e $66,40 \pm 3,48 \mathrm{~kg}$ para forwards e backs, respectivamente (AGAR-NEWMAN; GOODALE; KLIMSTRA, 2017). Em jogadoras brasileiras, foram encontradas massa corporal de 75,3 $\pm 10,6 \mathrm{~kg}$ para forwards e $61,8 \pm 7,0 \mathrm{~kg}$ para backs e estatura de $165,6 \pm 5,7 \mathrm{~m}$ para forwards e 162,9 $\pm 5,2 \mathrm{~m}$ para backs (AGAR-NEWMAN; GOODALE; KLIMSTRA, 2017). Esses achados foram semelhantes aos encontrados nesta investigação, sendo que as forwards apresentaram médias de

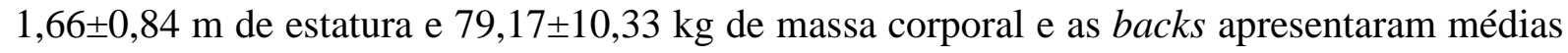
de $1,61 \pm 0,05 \mathrm{~m}$ de estatura e $62,23 \pm 5,84 \mathrm{~kg}$ de massa corporal, tendo essa variável com tamanho de efeito caracterizado como grande. De modo geral, estatura e massa corporal elevadas são relevantes no rugby para forwards principalmente devido à necessidade de realizar esforços de contato nessa posição. Além disso, a massa corporal deve ser reduzida em backs, pois estes devem exercer atividades de velocidade e agilidade durante o jogo.

A altura de salto é frequentemente avaliada no rugby, provavelmente porque a produção de potência é necessária para diversos movimentos específicos no jogo. Assim, DarrallJones, Jones e Till (2015) avaliaram o CMJ de jovens jogadores do sexo masculino de rugby union sub 21 e encontraram $47,1 \pm 3,6 \mathrm{~cm}$, resultados superiores aos jogadores do sexo masculino do presente estudo, que atingiram $34,2 \pm 8,14 \mathrm{~cm}$ (com diferença entre posições considerada como tamanho de efeito moderado), provavelmente devido aos atletas ingleses realizarem treinamento de potência com maior frequência, exibirem nível competitivo superior e média de idade ligeiramente inferior. No sexo feminino, o desempenho de neozelandezas no CMJ foi de 39,6 cm e 44,8 cm para forwards e backs, respectivamente, com diferença significante entre posições (GERODIMOS et al., 2008), corroborando as diferenças entre posições

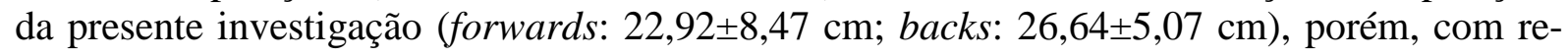
sultados superiores, provavelmente devido ao menor nível competitivo das atletas deste estudo. Nesse sentido, o CMJ deve ser analisado em diferentes níveis de jogadores de rugby, tendo em vista que a potência de membros inferiores é relevante nos saltos, nas acelerações e nas ações de projeção durante tackles. 
Além da potência de membros inferiores, o maior número de sprints realizados em jogos de rugby no sexo masculino foi registrado entre 11 e 20 m (AUSTIN, GABBETT; JENKINS, 2008). Assim, avaliar a capacidade de aceleração e a velocidade em provas de distâncias curtas, como $10 \mathrm{~m}$ e $20 \mathrm{~m}$, pode ser bastante relevante para a modalidade. Monica et al. (2016) avaliaram o tempo de sprint de atletas de rugby de nível universitário do sexo mascu-

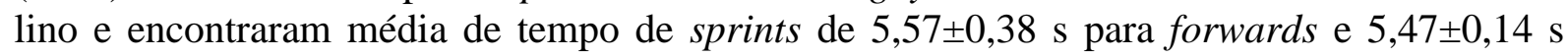
para backs percorrerem $40 \mathrm{~m}$, sem diferenças significativas entre posições, corroborando o presente estudo. Já em sprints de 10 e $20 \mathrm{~m}$, jogadores ingleses universitários de rugby union

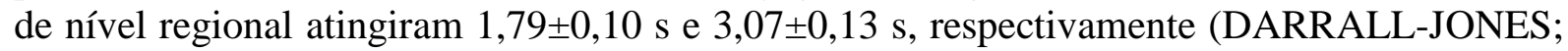
JONES; TILL, 2015), enquanto atletas do sexo masculino do presente estudo alcançaram $1,97 \pm 0,15 \mathrm{~s}$ em $10 \mathrm{~m}$ e $3,42 \pm 0,23 \mathrm{~s}$ em $20 \mathrm{~m}$, com diferenças entre posições nas duas distâncias com tamanho do efeito moderado, tempo superior pelo provável fato de que, além da média de idade inferior dos jogadores universitários, os jogadores do presente estudo apresentam quantidade de sessões de treinamento inferior.

O RAST é um modelo de esforço de natureza intermitente, com contribuições relativas das vias de fosforilação oxidativa, glicolítica e fosfágena de 38\%, $34 \%$ e $28 \%$, respectivamente (Milioni et al., 2017). Com efeito, ele tem sido empregado para avaliar a aptidão física de jogadores de futebol (ANDRADE et al., 2014), basquete (ARAUJO et al., 2014) e rugby (MEZZAROBA; TRINDADE; MACHADO, 2013), o que eleva sua relevância nas rotinas de avaliação de modalidades coletivas. Quanto aos valores de referência, membros das forças armadas obtiveram potência média no RAST de 590,62 $\pm 90,69 \mathrm{~W}$ (ZAGATTO et al., 2009). Entre jogadores amadores de rugby union de nível nacional que participam de eventos estaduais e nacionais, forwards obtiveram potência média de 468,5 $\pm 104,2 \mathrm{~W}$ e backs de 492,6 $\pm 119,0 \mathrm{~W}$, e potência de pico de $625,3 \pm 107,0 \mathrm{~W}$ em forwards e $643,1 \pm 171,9 \mathrm{~W}$ em backs (MEZZAROBA et al., 2013), sem diferenças significativas entre posições.

Tais resultados corroboram o presente estudo, o qual encontrou potência média em

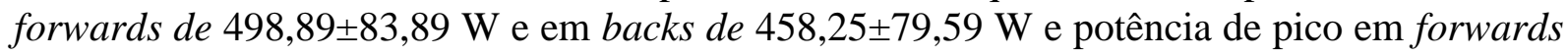
de $677,2 \pm 224,47 \mathrm{~W}$ e em backs de 578,26 $\pm 191,79 \mathrm{~W}$, esta variável com diferença entre posições e tamanho de efeito moderado. A semelhança provavelmente seja porque forwards e backs necessitam de deslocamentos rápidos e potentes em diferentes situações de jogo, como recuperação de bola, esquivas e ataques. Em relação aos valores relativos à massa corporal, os homens membros das forças armadas apresentaram potência (PRelMC) de $8,20 \pm 1,34 \mathrm{~W} \cdot \mathrm{kg}^{-1}$ (ZAGATTO et al., 2009), enquanto os jogadores de rugby do presente estudo apresentaram PRelMC $=5,29 \pm 1,22 \mathrm{~W} \cdot \mathrm{kg}^{-1}$. Essa diferença pode estar associada com o nível de aptidão física, bem como com o tipo de treinamento físico.

Reforça-se que sprints sucessivos são frequentes no rugby e que, além do RAST, outros procedimentos têm sido empregados para a avaliação da capacidade de sprints repetidos. Nesse cenário, destaca-se o teste Múltiplo de 5 (M5), por ser um procedimento que avalia com maior facilidade atletas de modalidades coletivas que exigem exercícios de alta intensidade e curta duração (BODDINGTON et al., 2001). Em atletas amadores de rugby do sexo masculino, no M5 foi encontrado desempenho de 642,9 $\pm 46,5 \mathrm{~m}$ para forwards e $686,8 \pm 36,6$ m para backs (SANT'ANNA; CASTRO, 2016), sem diferenças significantes entre posições, corroborando o presente estudo.

No presente estudo, foi investigada a correlação de variáveis antropométricas com variáveis da aptidão física. A massa corporal se correlacionou negativamente com a velocidade de sprint de $10 \mathrm{~m}(\mathrm{r}=-0,780)$ e $20 \mathrm{~m}(\mathrm{r}=-0,740)$ e não foram observadas correlações com estatura e sprints de 10 e 20 m, CMJ e SJ, como observado previamente (CREWTHER; MCGUIGAN; GILL., 2011), resultados que corroboram o presente estudo. Em atletas do sexo masculino de diferentes modalidades coletivas (rugby union, rugby league e basquete), o $s$ print de $5 \mathrm{~m}$ foi negativamente correlacionado à impulsão horizontal a partir do primeiro con- 
tato com o solo ( $\mathrm{r}=-0,640)$ (SLEIVERT; TAINGAHUE, 2004). Ainda, em universitários do sexo masculino, sprints de 10,15 e $30 \mathrm{~m}$ foram correlacionados inversamente com salto vertical ( $\mathrm{r}=-0,480, \mathrm{r}=-0,450$ e $\mathrm{r}=-0,770$, respectivamente) (CASTRO, 2010). Esses achados podem ser associados aos resultados do presente estudo, que observou correlação negativa significativa entre sprints e saltos verticais no sexo masculino (sprint de $10 \mathrm{~m}$ e SJ: $\mathrm{r}=-0,450$; sprint de 10 m e CMJ: r=-0,414; sprint de 20 m e SJ: r=-0,434; sprint de 20 m e CMJ: r=-0,413). Tais relações implicam a importância de associar exercícios de pliometria no planejamento de treino de potência em jogadores de rugby union que necessitam de alta velocidade de sprints.

Adicionalmente, jogadores de futebol australiano apresentaram correlação do tempo total de sprints repetidos e sprint de $20 \mathrm{~m}$ ( $\mathrm{r}=0,829)$ (WADLEY; LE ROSSIGNOL, 1998). No presente estudo, jogadores de rugby union do sexo masculino apresentaram correlação inversa entre potência média e sprint de $20 \mathrm{~m}(\mathrm{r}=-0,575)$, corroborando o estudo de Wadley e Le Rossignol (1998). Ainda nessa investigação, no sexo masculino, o M5 também foi correlacionado moderadamente com sprint de $20 \mathrm{~m}(\mathrm{r}=-0,511)$, sendo que o primeiro avalia a resistência relacionada à fadiga (DURANDT et al., 2006) durante um teste de resistência anaeróbia de múltiplos sprints. Também demonstrou estar relacionado às variáveis de potência (PRelMC no sexo masculino e CMJ no sexo feminino), provavelmente porque a capacidade de produção de potência pode reduzir a fadiga durante a sequência de sprints de curta distância. As variáveis de potência foram diferentes entre masculino e feminino, o que pode estar relacionado com as diferenças dos níveis de potência e força, visto que há diferenças nas especificidades estruturais (peso e estatura), morfológicas (quantidade de fibra tipo II), fisiológicas (capacidade recuperativa) e hormonais (níveis de testosterona) (LEITE et al., 2015).

Os resultados apresentados no presente estudo evidenciam a relação entre potência e velocidade e que variáveis antropométricas podem estar relacionadas com variáveis da aptidão física; porém, são dependentes do sexo, visto que houve correlações em diferentes variáveis nos sexos masculino e feminino. Apesar disso, investigações com sexo feminino acerca de correlações entre essas variáveis são escassas. Dessa forma, sugere-se que futuros estudos também investiguem a relação das variáveis no sexo feminino em diferentes faixas etárias e níveis competitivos.

\section{Conclusões}

No sexo masculino, forwards e backs diferenciaram-se nos testes SJ, CMJ, S10, S20, PP, PRelMC e VRAST. Além disso, as correlações se mostraram frequentemente distintas entre os sexos, e PR e IMC e CMJ explicam o desempenho no teste de capacidade anaeróbia em homens e mulheres, respectivamente. Jogadores amadores de rugby apresentam comportamentos diferentes entre sexos, visto que no sexo feminino não foram observadas diferenças significativas entre posições nos testes físicos, enquanto no sexo masculino forwards e backs são diferentes no SJ, CMJ, S10, S20, PP, PRelMC e VRAST. Ainda, houve correlações distintas nos sexos masculino e feminino, uma vez que PRelMC e CMJ explicam o desempenho no teste de capacidade anaeróbia em homens e mulheres, respectivamente. Tendo em vista que no Brasil o treinador e o preparador físico, na maioria dos clubes amadores brasileiros, ocupam-se das equipes dos dois sexos, é fundamental a compreensão das reais diferenças entres homens e mulheres no que se refere aos aspectos físicos. Nesse sentido, treinadores de rugby amador que conhecem as variáveis que caracterizam seus jogadores e suas diferenças entre posições podem tornar seus programas de treinos eficientes e mais específicos. 


\section{Referências}

AGAR-NEWMAN, D. J.; GOODALE, T.; KLIMSTRA, M. Anthropometric and physical qualities of international level female rugby sevens athletes based on playing position. Journal of Strength and Conditioning Research, Canada, v. 31, n. 5, p. 1346-1352, maio 2017.

ANDRADE, V. L.; PEREIRA SANTIAGO, P. R.; KALVA FILHO, C. A.; CAMPOS, E. Z.; PAPOTI, M. Reproducibility of running anaerobic sprint test (RAST) for soccer players. The Journal of Sports Medicine and Physical Fitness, São Paulo, v. 56, n. 1, p. 34-38, nov. 2014.

ARAUJO, G.G.; MANCHADO-GOBATTO, F. B.; PAPOTI, M.; CAMARGO, B. H. F.; GOBATTO, C. A. Anaerobic and aerobic performances in elite basketball players. Journal of Human Kinetics, Campinas, v. 42, n. 1, p. 137-147, set. 2014.

AUSTIN, D.; GABBETT, T., JENKINS, D. The physical demands of Super 14 rugby union. Journal of Science and Medicine in Sport, Australia, v. 14, n. 3, p. 259-263, maio 2011.

BAKER DG. Comparison of strength levels between players from within the same club that were selected vs. not selected to play in the grand final of the National Rugby League competition. Journal of Strength and Conditioning Research, Australia, v. 31, n. 6, p. 1461-1467, jun. 2017.

BODDINGTON, M. K.; LAMBERT, M. I.; GIBSON, A. S. C.; NOAKES, T. D. Reliability of a 5-m multiple shuttle test. Journal of Sports Science, Newlands, v. 19, n. 3, p. 223-228, mar. 2001.

CASTRO, M.L.A. Relações entre diferentes manifestações de força explosiva em estudantes universitários do sexo masculino. 2010. 31f. Dissertação (Mestrado em Ciências do Desporto) - Faculdade de Ciências Sociais e Humana, Departamento de Ciências do Desporto, Universidade da Beira Interior, Covilhã, 2010.

COMFORT, P.; GRAHAM-SMITH, P.; MATTHEWS, M. J.; BAMBER, C. Strength and power characteristics in English elite rugby league players. Journal of Strength and Conditioning Research, United Kingdom, v. 25, n. 5, p. 1374-1384, maio 2011.

CREWTHER, B.T.; MCGUIGAN, M.R.; GILL, N.D. The ratio and allometric scaling of speed, power, and strength in elite male rugby union players. Journal of Strength and Conditioning Research, New Zeland, v. 25, n. 7, p. 1968-1975, jul. 2011.

CUNNINGHAM, D.; SHEARER, D. A.; DRAWER, S.; EAGER, R.; TAYLOR, N.;

COOK, C.; KILDUFF L. P. Movement demands of elite U20 international rugby union players. PLoS One, Wales, v. 11, n. 4, abr. 2016.

DARRALL-JONES, J. D.; JONES, B.; TILL, K. Anthropometric and physical profiles of english academy rugby union players. Journal of Strength and Conditioning Research, United Kingdom, v. 29, n. 8, p. 2086-2096, ago. 2015. 
DEUTSCH, M. U.; KEARNEY, G. A.; REHRER, N. J. Time-motion analysis of professional rugby union players during match-play. Journal of Sports Science, United Kingdom, v. 25, n. 4, p. 461-472, fev. 2007.

DURANDT, J.; TEE, J.C.; PRIM, S.K.; LAMBERT, M.I. Physical fitness components associated with performance in a multiple-sprint test. Internatinal Journal of Sports Physiology and Performance, South Africa, v. 1, n. 1, p. 150-160, jun. 2006.

DUTHIE, G. A framework for the physical development of elite rugby union players. International Journal of Sports Physiology and Performance, Australia, v. 1, n. 1, p. 2-13, mar. 2006.

DUTHIE, G.; PYNE, D.; HOOPER, S. Applied physiology and game analysis of rugby union. Sports Medicine, Australia, v. 33, n. 13, p. 973-991, 2003.

FULLER, C. W.; BROOKS, J. H. M.; CANCEA, R. J.; HALL, J.; KEMP, S. P. T. Contact events in rugby union and their propensity to cause injury. British Journal of Sports Medicine, United Kingdom, v. 41, n. 12, p. 862-867, dez. 2007.

GERODIMOS, V.; ZAFEIRIDIS, A.; PERKOS, S.; DIPLA, D.; MANOU, V.; KELLIS, S. The contribution of stretch-shortening cycle and arm-swing to vertical jumping performance in children, adolescents, and adult basketball players. Pediatric Exercise Science, Greece, v. 20, n. 4, p. 379-389, nov. 2008.

HOHENAUER, E.; RUCKER, A. M.; CLARYS, P.; KÜNG, U. M.; STOOP, R.; CLIJSEN, $\mathrm{R}$. Anthropometric and performance characteristics of the German rugby union $7 \mathrm{~s}$ team. The Journal of Sports Medicine and Physical Fitness, Switzerland, v. 57, n. 12, p. 1633-1641, 2017.

HAFF, G. G.; NIMPHIUS, S. Training principles for power. National Strength Conditioning Association, Australia, v. 34, n. 6, p. 1-12, dez. 2012.

HRYSOMALLIS, C. Neck muscular strength, training, performance and sport injury risk: a review. Sports Medicine, Australia, v. 46, n. 8, p. 1111-1124, ago. 2016.

JARVIS, S.; SULLIVAN, L. O.; DAVIES, B.; WILTSHIRE, H.; BAKER, J. S. Interrelationships between measured running intensities and agility performance in sub-elite rugby union players. Research in Sports Medicine, United Kingdom, v. 17, n. 4, p. 217-230, 2009.

JONES, M.R.; WEST, D.J.; CREWTHER, B.T.; COOK, C.J.; KILDUFF, L.P. Quantifying positional and temporal movement patterns in professional rugby union using global positioning system. Europen Journal of Sports Science, United Kingdom, v. 15, n 6, p. 488-496, 2015.

KOBAL, R.; NAKAMURA, F. Y.; MORAES, J. E.; COELHO, M.; KITAMURA, K.; CAL ABAD, C. C.; PEREIRA, L. A.; LOTURCO, I. Physical performance of Brazilian rugby players from different age-categories and competitive levels. Journal of Strength and Conditioning Research, Londrina, v. 30, n. 9, p. 2433-2439, set. 2016. 
LEITE, M. A. F. J.; GUEDES, J. M.; ANGELOTTI, L. C. Z.; MOTTA, G.R.; MENDES, E.L. Análise do pico de potência de membros superiores em jogadores amadores de rugby. Conexões, Uberaba, v. 13, n. 2, p. 15-28, abr./jun. 2015.

MARKOVIC, G.; DIZDAR, D.; JUKIC, I.; CARDINALE, M. Reliability and factorial validity of squat and countermovement jump tests. Journal of Strength and Conditioning Research, Croatia, v. 18, n. 3, p. 551-555, ago. 2004.

MEZZAROBA, P.V.; TRINDADE, C. F.; MACHADO, F. A. Indicadores fisiológicos e antropométricos de uma amostra de atletas brasileiros de rugby. Revista Brasileira de Ciências do Esporte, Maringá, v. 35, n. 4, p. 1021-1033, out./dez. 2013.

MILIONI, F.; ZAGATTO, A. M.; BARBIERI, R. A.; ANDRADE, V. L.; SANTOS, J. W.; GOBATTO, C. A.; SILVA, A. S. R.; SANTIAGO, P. R. P.; PAPOTI, M. Energy systems contribution in the running-based anaerobic sprint test. International Journal of Sports Medicine, Rio Claro, v. 38, n. 3, p. 226-232, mar. 2017.

MOIR, G.; BUTTON, C.; GLAISTER, M.; STONE, M. H. Influence of familiarization on the reliability of vertical jump and acceleration sprinting performance in physically active men. Journal of Strength and Conditioning Research, Scotland, v. 18, n. 2, p. 276-280, maio 2004.

MONICA, M. B.; FUKUDA, D. H.; MIRAMONTI, A. A.; BEYER, K. S.; HOFFMAN, M. W.; BOONE, C. H.; TANIGAWA, S.; WANG, R.; CHURCH, D. D.; STOUT, J. R.; HOFFMAN, J. R. Physical differences between forwards and backs in American collegiate rugby players. Journal of Strength and Conditioning Research, Florida, v. 30, n.9, p. 23821291, set. 2016.

NAKAMURA, F. Y.; PEREIRA, L. A.; MORAES, J. E.; KOBAL, R.; KITAMURA, K.; CAL ABAD, C. C.; TEIXEIRA VAZ, L. M.; LOTURCO, I. Physical and physiological differences of backs and forwards from the Brazilian National rugby union team. The Journal of Sports Medicine and Physical Fitness, São Paulo, v. 1, n. 1, p. 1-24, nov. 2016.

NICHOLAS, C. W. Anthropometric and physiological characteristics of rugby union football players. Sports Medicine, United Kingdom, v. 23, n. 6, p. 375-396, jun. 1997.

PINHEIRO, E. S.; MIGLIANO, M.; BERGMANN, G. G.; GAYA, A. Desenvolvimento do rugby brasileiro: panorama de 2009 a 2012. Revista Mineira de Educação Física, Porto Alegre, v. 29, n. 9, p. 990-995, ed. esp., 2013.

QUARRIE, K. L.; HANDCOCK, P.; WALLER, A. E.; CHALMERS, D. J.; TOOMEY, M. J.; WILSON, B. D. The New Zealand rugby injury and performance project III: Anthropometric and physical performance characteristics of players. British Journal of Sports Medicine, New Zeland, v. 29, n. 4, p. 263-270, dez. 1995.

RHEA, M.R. Determining the magnitude of treatment effects in strength training research through the use of effect size. Journal of Strength and Conditioning Research. United States, v. 18, n. 4, p. 918-920, 2004. 
ROE, G. A. B.; DARRALL-JONES, J. D.; TILL, K. T.; JONES, B. Preseason changes in markers of lower body fatigue and performance in: young professional rugby union players. European Journal of Sports Science, United Kingdom, v. 16, n. 8, p. 1-8, nov. 2016.

SANT'ANNA R. Determinação da potência aeróbia em jogadores amadores de rugby union XV a partir de testes de campo. 2015. 61f. Dissertação (Mestrado em Ciências do Movimento Humano) - Programa de Pós-Graduação em Ciências do Movimento Humano, Universidade Federal do Rio Grande do Sul, Porto Alegre, 2015.

SANT'ANNA, R.; CASTRO, F.A. Aerobic power and field test results of amateur 15-a-side rugby union players. The Journal of Sports Medicine and Physical Fitness, Porto Alegre, jul. 2016.

SLEIVERT, G.; TAINGAHUE, M. The relationship between maximal jump-squat power and sprint acceleration in athletes. Europen Journal of Applied Physiology, Canada, v. 91, n. 1, p. 46-52, jan. 2004.

SOUSA, N. M. F.; STINGUEL, H.; MAIRINK, R. S.; BAIA, D. P.; BERTUCCI, D. R.; MARTINS, R. A. S. Perfil antropométrico e aeróbio de jogadoras de uma equipe de rugby sevens: diferenças entre posições táticas. Revista Brasileira de Prescrição e Fisiologia do Exercício, Vítória, v. 10, n. 58, p. 282-289, mar./abr. 2016.

WADLEY, G.; LE ROSSIGNOL, P. The relationship between repeated sprint ability and the aerobic and anaerobic energy systems. Journal of Science and Medicine in Sport, v. 1, n. 2, p. 100-110, jun. 1998.

WOOD, D. J.; COUGHLAN, G.; DELAHUNT, E. Fitness profiles of elite adolescent irish rugby union players. Journal of Strength and Conditioning Research, Ireland, v. 32, n. 1, p. 105-112, 2018.

ZAGATTO, A. M.; BECK, W. R.; GOBATTO, C. A. Validity of the running anaerobic sprint test for assessing anaerobic power and predicting short-distance performances. Journal of Strength and Conditioning Research, Campo Grande, v. 23, n. 6, p. 1820-1827, set. 2009.

Recebido em: $18 / 10 / 2017$

Revisado em: 28/05/2018

Aprovado em: 18/07/2018

Endereço para correspondência:

camilaborges1210@gmail.com

Camila Borges Müller

Universidade Federal de Pelotas, Escola Superior de Educação Física

Rua Luís de Camões, 625

Três Vendas

96055-630 - Pelotas, RS - Brasil 